\title{
METODOLOGÍA PARA LA DESCRIPCIÓN DE GÉNEROS EN EL MARCO DE LA LINGÜÍSTICA SISTÉMICO-FUNCIONAL: SU \\ ADAPTACIÓN AL ESPAÑOL
}

\author{
METHODOLOGY FOR GENRE DESCRIPTION IN THE FRAME OF \\ SYSTEMIC-FUNCTIONAL LINGUISTICS: AN ADAPTATION TO \\ SPANISH
}

Estela Inés Moyano (UNGS/UNaB/UFLO)

estelaimoyano@gmail.com

\begin{abstract}
RESUMEN: En el presente trabajo se abordará la metodología para la descripción de géneros en el marco de la lingüística sistémico-funcional (LSF) adaptada al español, con el propósito de aportar a campos como el análisis del discurso y la aplicación a la enseñanza de la lectura y la escritura basada en géneros en esa lengua. Con ese fin, se considera la LSF como una lingüística aplicable (Halliday, 2008), lo que la posiciona en relación con el contexto, como modo de intervención en prácticas sociales. La base teórico-metodológica que se utiliza es la propuesta de Martin (1992a), desarrollada luego por el propio Martin y sus discípulos a lo largo del tiempo y hasta la actualidad. Dado que ésta ha sido elaborada para el inglés, se hacen adaptaciones al español cuando resulta necesario, a partir de investigaciones realizadas en la última década.
\end{abstract}

PALABRAS CLAVE: descripción; discurso; lingüística sistémico-funcional; español.

ABSTRACT: The aim of this paper is to approach the methodology for description of genres in the frame of systemic-functional linguistics (SFL) in Spanish. The purpose of this work is to contribute to fields of discourse analysis and its application to genre-based pedagogies to teaching reading and writing in that language. To do this, SFL is treated as an appliable linguistics (Halliday, 2008), related to its context, as a mode of intervention in critical social practices. The theoretical and methodological base utilized is from Martin (1992a), developed afterwards by Martin and his disciples over time up to nowadays. As this proposal has been established for English, here it is adapted to Spanish when necessary, departing from research done in the last decade.

KEY-WORDS: description; discourse; systemic-functional linguistics; Spanish. Introducción 
La lingüística sistémico-funcional (LSF) es considerada por Halliday (2008) una lingüística aplicable. Esta denominación hace referencia a una lingüística que da cuenta de la realidad social, en el sentido de que pone el lenguaje en su contexto y, al mismo tiempo, constituye un modo de intervención en prácticas sociales críticas (HALLIDAY, 2003 [1993]). Dos de esas actividades son el análisis del discurso y su aplicación a procesos de enseñanza con el propósito de ampliar las habilidades o competencias en lectura y escritura de textos disciplinares. Desarrollar las competencias en el manejo del lenguaje de las disciplinas aumenta la capacidad de construir conocimiento (BERNSTEIN, 2000), de manera que efectivamente se produce un impacto social relevante. Halliday (2004 [1993]) explica que, debido a sus características especialmente desarrolladas, enseñar el lenguaje científico evita la alienación de los estudiantes frente a un texto disciplinar, es decir, permite la construcción de conocimiento científico. Además, este uso particular del lenguaje se extiende a otros ámbitos sociales, como el de la política, la administración, los medios de comunicación. Martin (1993) advierte que quienes no accedan a este tipo de discurso quedarán excluidos de la participación en procesos sociales relevantes.

En América Latina existe consenso generalizado acerca de la necesidad de la enseñanza de la lectura y la escritura de textos académicos y profesionales a nivel universitario (MOYANO, 2004; CARLINO, 2005; NAVARRO, 2017, entre muchos otros). Pero, además, es necesario llevar a cabo este trabajo en niveles educativos anteriores, como la escuela secundaria y aun la primaria (CHRISTIE; MARTIN, 1997; FEEZ, 2006; CHRISTIE, 2012; ROSE; MARTIN, 2012; DE SILVA JOYCE; FEEZ, 2012; DREWIANKA; JONES, 2012; MOYANO, 2013; HENRÍQUEZ; CARMONA; QUINTEROS; GARRIDO, 2018).

El propósito de este artículo es dar cuenta de la metodología de análisis de género, registro y discurso basada en la lingüística sistémico-funcional adaptada al español, aplicable a géneros de los ámbitos sociales mencionados. Se considera que resulta necesario plantear un recurso metodológico para el análisis del discurso en este marco, que puede ser aplicado para la enseñanza de la lectura y la escritura así como también para otros propósitos de intervención social.

Para ello, se dará cuenta en primer lugar del marco teórico en el que se basa la propuesta metodológica, para luego describirla mediante su aplicación. Se utilizarán como ejemplo textos sencillos, tomados de un manual de nivel secundario que fue utilizado muchos años en escuelas universitarias. Cabe señalar, sin embargo, que se trata de un método ya utilizado para el análisis de géneros en ciencias y humanidades de niveles más avanzados, 
instanciados en lengua española (MOYANO, 2010a; 2014; 2015; 2019; en prensa; VIDAL LIZAMA, 2020; VIDAL LIZAMA; LEIVA, en prensa; VIDAL LIZAMA; MONTES, en prensa). La utilización de textos sencillos en esta ocasión facilitará la explicación metodológica.

\section{Marco teórico}

La LSF constituye una teoría del lenguaje utilizado para la vida social. Esto es, se trata de un marco que se propone explicar cómo el lenguaje lleva a cabo las actividades que los humanos desplegamos. El lenguaje ha sido definido como el potencial de significados disponibles en una cultura que se organizan como sistemas de opciones en un contexto social dado y se actualizan en la construcción de mensajes (HALLIDAY, 1982 [1978], MATTHIESSEN; HALLIDAY, 2009).

Así, el lenguaje se concibe en relación con su contexto, entendiendo que este se realiza en el lenguaje, mientras el lenguaje, a su vez, lo construye. Esta relación de mutua dependencia se establece entre dos sistemas semióticos, ambos productores de significado. El contexto es un sistema semiótico connotativo, que carece de plano de realización, de manera que necesita de otro sistema, denotativo, para manifestarse (MARTIN, 1992a; 2014; MARTIN; ROSE, 2007). En el caso de los textos que son objeto de análisis en este trabajo, el sistema semiótico denotativo que realiza los significados del contexto es el lenguaje.

Tanto el contexto como el lenguaje son entendidos aquí, siguiendo a Martin (2014) como sistemas semióticos estratificados que se grafican a través de círculos supervinientes, es decir, cotangenciales (Figura 1). Esto significa que la relación entre contexto y lenguaje es intrínseca y que el contexto no es material sino una construcción teórica (HALLIDAY, 2007 [1991]). 


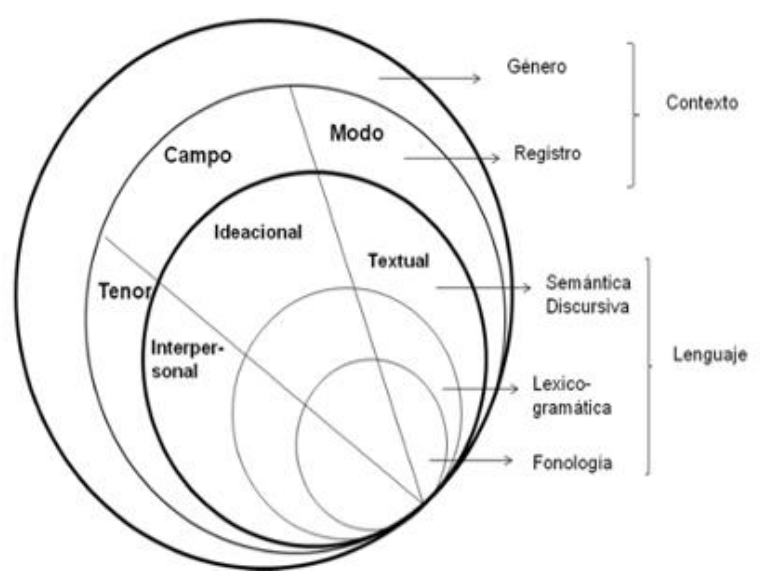

Figura 1: Contexto y lenguaje estratificados y organizados por metafunción Fuente: MARTIN; ROSE, 2007

En cuanto a la estratificación, el contexto se organiza en género y registro. El género puede ser definido como combinación de patrones de campo, tenor y modo que se interrelacionan y despliegan en una estructura esquemática que le permite lograr un propósito social específico (MARTIN, 2014; MARTIN; ROSE, 2008). El registro es la denominación que Martin (2014) utiliza como cobertura para las variables de campo, tenor y modo definidas como contexto por Halliday (1982 [1978]). En cuanto al lenguaje, se estratifica en semánticadiscursiva, lexicogramática y fono-grafía, que tienen diferentes unidades de análisis: la semántica-discursiva analiza el texto; la lexicogramática, la cláusula; y la fono-grafía las unidades tonales y los grafemas. Asimismo, la teoría considera diferentes rangos en cada estrato del lenguaje, relevantes para la descripción y el análisis de textos.

En otra dimensión de análisis, el lenguaje se organiza en metafunciones. Esto significa que genera sistemas de diferente tipo de significado: significados ideacionales, interpersonales y textuales, que se co-relacionan, a su vez, con las variables del registro, denominadas campo, tenor y modo. Dicho de manera muy simplificada, el campo, que se realiza mediante la metafunción ideacional del lenguaje, construye mundo; el tenor, que se realiza mediante la metafunción interpersonal, relaciones entre los participantes de la interacción; el modo, mediante la metafunción textual, construye texto, de manera de permitir combinaciones entre los otros dos tipos de significado.

En cuanto al análisis de género, Martin (2014) llama la atención sobre la necesidad de tener en cuenta su realización en el registro para luego analizar los significados del estrato semántico-discursivo, teniendo en cuenta su realización en la lexicogramática, sin saltar estratos pasando del género al discurso o a la lexicogramática. 
Para ese trabajo, ha habido gran desarrollo de la teoría, aunque aquí solo podamos dar cuenta de algunos trabajos. Con relación al lenguaje, los significados generados por el estrato fonológico han sido estudiados por Halliday y Greaves (2008); los producidos en la lexicogramática, por Halliday (HALLIDAY; MATTHIESSEN, 2014) y los generados en el discurso por Martin (MARTIN, 1992a; MARTIN; WHITE, 2005; MARTIN; ROSE, 2007). Con respecto al género, ha sido estudiado por Martin (MARTIN, 1992a; MARTIN; ROSE, 2008) y en relación con el registro hay estudios de Martin que avanzan desde 1992a en adelante. Pero la teoría sigue en movimiento, y hay estudios recientes sobre registro (MARTIN, 2007a; b; 2020; MARTIN; MATRUGLIO, 2020; DORAN; MARTIN, 2021) y sobre semántica-discursiva (HAO, 2020; 2021).

A los fines de analizar género, Martin ha propuesto una metodología (MARTIN; ROSE, 2007; 2008; MARTIN, 2009) que se procura adaptar en este trabajo al español, tomando algunos desarrollos que abordan la descripción de esta lengua en diferentes estratos (QUIROZ, 2008; 2015; 2017; MOYANO, 2010b; 2016, 2021; MARTIN; QUIROZ; WANG; ZHU, en prensa; OTEIZA, 2017; 2021 OTEIZA, HENRÍQUEZ; CANELO, 2018).

\section{La propuesta metodológica}

Para el análisis del discurso en el marco de la teoría de género en la LSF, se parte del análisis de cada texto, a fin de determinar su estructura esquemática así como los recursos semántico-discursivos que contribuyen a su realización. En este proceso, se trata de explicar, teniendo en cuenta los sistemas a partir de los cuales se realiza cada elección para la elaboración del texto, qué significados construyen estos recursos en su desarrollo y el propósito social que la unidad de discurso analizada persigue.

El primer paso a dar en el análisis de un texto como instancia de un género es prepararlo mediante su separación en cláusulas. Si bien no se hará el análisis a partir de estas unidades, este ejercicio permite observar el texto en detalle para poner en evidencia los elementos que funcionan tanto en el campo (DORAN; MARTIN, 2021) como en el estrato semántico-discursivo y su realización en el léxico-gramatical (MARTIN, 2009; MARTIN; ROSE, 2007; HAO, 2020, 2021). La opción de mirar el texto desde el registro para luego centrarse en el nivel de mayor abstracción del lenguaje, el estrato semántico-discursivo, haciendo solo algunas referencias al estrato lexicogramatical, se toma por tres razones: la existencia de motivos semánticos, tipos de significados que tienen diferentes realizaciones a lo largo de los sistemas gramaticales; la existencia de la metáfora gramatical, proceso por el cual 
una estructura requiere más de un nivel de interpretación; y, finalmente, la necesidad de dar cuenta del texto como unidad estructural que excede los límites de la léxico-gramática (MARTIN, 1992a). Esto significa que, en la propuesta metodológica que aquí se presenta, el análisis no se realiza cláusula a cláusula, sino tomando como unidad el texto (MARTIN; ROSE, 2007).

Para la segmentación del texto, se toman los conceptos de cláusula, complejo clausular, cláusula incrustada y cláusula incluida (HALLIDAY; MATTHIESSEN, 2014), que se señalan según se muestra en el Cuadro 1.

\begin{tabular}{|l|l|}
\hline Símbolo & Unidad \\
\hline$I / /$ & Complejo clausal \\
\hline$/ /$ & Cláusula \\
\hline$[[\quad]]$ & Cláusula incrustada \\
\hline$<\quad>>$ & Cláusula incluida \\
\hline
\end{tabular}

Cuadro 1: Símbolos utilizados en la segmentación del texto en cláusulas

Fuente: adaptado de Halliday y Matthiessen, 2014.

Los textos que se utilizan aquí como ejemplos (Tablas 1 y 2) son extraídos de un manual de biología (VILEÉ, 1975), que, como ya se indicó, fue utilizado por mucho tiempo en escuelas secundarias, particularmente en algunas dependientes de universidades. En los Cuadros 2 y 3 se los presenta segmentados en cláusulas para iniciar el análisis. Las cláusulas dependientes e independientes se señalan con una letra del abecedario. Cabe aclarar que el criterio tomado para la separación en cláusulas en español es el criterio ideacional, entre los tres establecidos por Martin, Matthiessen y Painter (2010). Este criterio señala que la presencia de un Proceso indica la existencia de una cláusula. Los Procesos, que se realizan mediante grupos verbales que en español incluyen los pronombres clíticos (QUIROZ, 2017), se indican en negrita. En algunos casos en español, los Procesos están elididos porque se recuperan de cláusulas anteriores, de manera que la unidad se identifica claramente (Tabla 1: h, i, k). 


\begin{tabular}{|l|l|}
\hline a. & Un músculo esquelético típico es una masa alargada de tejido,// \\
\hline b. & formada por millones de fibras musculares individuales unidas por otras de tejido conjuntivo./// \\
\hline c. & Toda la estructura está rodeada por una vaina elástica y lisa de tejido conjuntivo,// \\
\hline d. & $\begin{array}{l}\text { de modo que puede moverse sobre los músculos y otras estructuras adyacentes con un mínimo de } \\
\text { fricción./// }\end{array}$ \\
\hline e. & Los dos extremos de un músculo están unidos, generalmente, a dos huesos distintos,// \\
\hline f. & $\begin{array}{l}\text { aunque unos pocos pasan de un hueso a la piel, o de una parte de la piel a otra, como en caso de los } \\
\text { músculos faciales utilizados para la palabra y la expresión./// }\end{array}$ \\
\hline g. & $\begin{array}{l}\text { El extremo [[que permanece relativamente fijo cuando el músculo se contrae]], se denomina inserción de } \\
\text { origen;// }\end{array}$ \\
\hline h. & [[el que se mueve]] inserción terminal, // \\
\hline i. & y la parte gruesa comprendida entre ambos, vientre./// \\
\hline j. & La inserción de origen del músculo bíceps está en el hombro,// \\
\hline k. & y la terminal en el radio, en el antebrazo;// \\
\hline l. & cuando el bíceps se contrae,// \\
\hline m. & el hombro permanece fijo// \\
\hline n. & y el codo se flexiona./// \\
\hline
\end{tabular}

Tabla 1: Músculos esqueléticos

Fuente: Villée, 1975, p. 386-387, destaques del autor

\begin{tabular}{|l|l|}
\hline a. & $\begin{array}{l}\text { Las Chlamydomonas, «que se encuentran en charcos, lagos y sobre la tierra húmeda,» poseen una célula } \\
\text { vegetativa [[que tiene dos flagelos]] // }\end{array}$ \\
\hline b. & y está [[protegida por una gruesa pared celulósica]]./// \\
\hline c. & La célula puede reproducirse asexualmente por división,// \\
\hline d. & formando de dos a ocho zoosporas dentro de la pared celulósica./// \\
\hline e. & Estas se liberan por rotura de la pared celular del progenitor// \\
\hline f. & y se alejan,// \\
\hline g. & constituyendo plantas independientes./// \\
\hline h. & Ocasionalmente, se realiza la reproducción sexual:// \\
\hline i. & la célula progenitora se divide para formar de ocho a treinta y dos células más pequeñas, las gametas,// \\
\hline j. & que se parecen a las zoosporas y a los individuos adultos// \\
\hline k. & pero son de menor tamaño./// \\
\hline l. & Dos de estas gametas se fusionan,// \\
\hline m. & comenzando por el extremo en [[el que nacen los flagelos]],// \\
\hline n. & para formar una cigota./// \\
\hline ñ. & Esta, al principio, tiene cuatro flagelos, dos de cada gameta,// \\
\hline o. & pero con el tiempo los pierde./// \\
\hline p. & La célula se redondea,// \\
\hline q. & segrega una gruesa pared celular// \\
\hline r. & y es capaz de sobrevivir largos períodos en un ambiente desfavorable./// \\
\hline s. & Cuando este se torna de nuevo favorable,// \\
\hline t. & la cigota sufre la meiosis// \\
\hline u. & y forma cuatro células./// \\
\hline v. & La pared celular se resquebraja// \\
\hline w. & dejando en libertad a las células,// \\
\hline x. & que emiten flagelos// \\
\hline y & y se transforman en plantas independientes./// \\
\hline & \\
\hline
\end{tabular}

Tabla 2: Las Chlamydomonas

Fuente: Villée, 1975, p. 192, destaques del autor.

Interesa trabajar con estos dos textos diferentes para poder mostrar aspectos que son relevantes a la hora de distinguir entre géneros. De todas maneras, por razones de espacio, no será posible un despliegue en tablas de todo el análisis, como sería deseable.

Luego de la preparación del texto mediante su separación en cláusulas, se realiza una primera mirada para considerar el registro, a fin de seguir el principio de la LSF que toma el 
texto en su contexto. Como se ve en la Figura 1, se tiene en cuenta la actividad social que realizan (campo), la interacción con la audiencia destinataria del mensaje (tenor) y sus características de texto escrito (modo). En principio, si se atiende al campo, y como se verá en detalle enseguida, se observa que ambos textos presentan diferencias en el tipo de actividades que despliegan: el primero, actividades estáticas; mientras el segundo, actividades dinámicas (DORAN; MARTIN, 2021). En cuanto al tenor, se observan en ambos textos escasas interacciones con el destinatario: se trata de textos monológicos, que, como se mostrará, apenas presentan algunos signos de valoración para lograr su propósito. Finalmente, si se considera el modo, ambos son textos escritos, constitutivos de la actividad, que presentan alto grado de tecnicalidad y distancia semiótica, con lo que se ha dado en llamar independencia del contexto (MARTIN, 1992a; MARTIN; ROSE, 2007; MARTIN; MATRUGLIO, 2020).

A continuación, se procura identificar el género que cada texto instancia, buscando reconocer su propósito social y las etapas que lo componen (MARTIN; ROSE, 2007). Como punto de partida, se observan las características lingüísticas salientes en el texto, las que aparecen en primer plano, y su coincidencia con los rasgos relevantes de un género entre los descriptos en este marco (MARTIN; ROSE, 2008). Se tiene en cuenta para ello el despliegue del campo del registro, teniendo en cuenta su realización a través de la metafunción ideacional en el estrato semántico-discursivo, observando por un lado las entidades en juego, por otro las actividades y los tipos de procesos que las realizan en el estrato lexicogramatical, así como también las conjunciones, prestando atención a si tienen relevancia las relaciones temporales y/o las causales, si son más relevantes las relaciones lógicas externas o las internas.

Hao (2015; 2020), propone la figura como configuración orbital de entidades, actividades y otros elementos. La figura es un elemento semántico-discursivo que se realiza lexicogramaticalmente a través de una cláusula (HAO, 2021). Existen, en primera instancia, dos tipos de figura: las figuras de ocurrencia (occurrence figures) y las figuras de estado (state figures). Las primeras se realizan gramaticalmente mediante procesos materiales o de comportamiento, mientras las segundas, mediante procesos relacionales, que vinculan entre sí entidades o entidades con sus cualidades o que meramente señalan su existencia (HAO, 2020). Desde una perspectiva del campo, las figuras de ocurrencia realizan actividades, mientras que las de estado facilitan las relaciones taxonómicas (DORAN; MARTIN, 2021).

En este sentido, los textos que presentamos en las Tablas 1 y 2 se distinguen claramente: el Texto 1 presenta un predominio de figuras de estado, mientras el Texto 2 un predominio de figuras de ocurrencia. En cuanto a las relaciones lógicas, una primera 
observación permite hipotetizar que en los dos casos se trata de conexiones externas, pero que en el primer texto son mayormente de adición mientras en el segundo se combina la adición con la causalidad. Estos dos rasgos rápidamente orientan, atendiendo a los géneros elementales descriptos para las ciencias naturales y físicas (MARTIN; ROSE, 2008), a hipotetizar que el Texto 1 se encuentra entre los informes, mientras que el Texto 2 entre las explicaciones.

Si se observan las relaciones taxonómicas, que Martin y Rose (2007) ubicaban como uno de los subsistemas de IDEACIÓN y que Doran y Martin (2021) ubican en el campo del registro, se observa con claridad que en el Texto 1 se presenta una entidad, un músculo esquelético típico. A continuación, se presentan sus características generales en las figuras (a) a (f) (es una masa alargada de tejido, [está] formada por..., está [[rodeada...]]). Luego de presentarlo en la figura (a) (versalitas) y de caracterizarlo en (b) a (f), se enumeran sus partes (subrayado) en las figuras (g) a (i), en relación de meronimia, partes a las que se les da nombre (cursiva) (Tabla 3).

\begin{tabular}{|l|l|}
\hline a. & UN MÚSCULO ESQUELÉTICO TÍPICO es una masa alargada de tejido,// [...] \\
\hline g. & El extremo [[que permanece relativamente fijo cuando el músculo se contrae]], se denomina inserción de \\
origen;//
\end{tabular}

Tabla 3: Relaciones taxonómicas en Músculos Esqueléticos

Fuente: Villée, 1975, p. 386, destaques del autor

Esta primera mirada al texto sugiere que se trata de un informe composicional, cuyas características se comprobarán a medida que se observen los otros recursos en juego.

En cuanto a los recursos del sistema de CONEXIÓN (HAO, 2015), muestran que la mayoría de las relaciones entre figuras es de adición (Tabla 5). En general, son pocas las conjunciones explícitas, pero bastan para generar la expectativa propia del género. 


\begin{tabular}{|l|l|l|}
\hline & a. & Un músculo esquelético típico es una masa alargada de tejido, \\
\hline & b. & $\begin{array}{l}\text { formada por millones de fibras musculares individuales unidas por otras de tejido } \\
\text { conjuntivo. }\end{array}$ \\
\hline consec & c. & Toda la estructura está rodeada por una vaina elástica y lisa de tejido conjuntivo, \\
\hline & d. & $\begin{array}{l}\text { de modo que puede moverse sobre los músculos y otras estructuras adyacentes con un } \\
\text { mínimo de fricción. }\end{array}$ \\
\hline difer & e. & Los dos extremos de un músculo están unidos, generalmente, a dos huesos distintos, \\
\hline & f. & $\begin{array}{l}\text { aunque unos pocos pasan de un hueso a la piel, o de una parte de la piel a otra, como en } \\
\text { el caso de los músculos faciales utilizados para la palabra y la expresión. }\end{array}$ \\
\hline [adic] & g. & $\begin{array}{l}\text { El extremo que permanece relativamente fijo cuando el músculo se contrae, se } \\
\text { denomina inserción de origen; }\end{array}$ \\
\hline adic & h. & [y] el que se mueve inserción terminal, \\
\hline \multicolumn{1}{|l|}{} & i. & y la parte gruesa comprendida entre ambos vientre. \\
\hline adic & j. & La inserción de origen del músculo bíceps está en el hombro, \\
\hline & k. & y la terminal en el radio, en el antebrazo; \\
\hline & l. & cuando el bíceps se contrae, \\
\hline adic & m. & el hombro permanece fijo \\
\hline
\end{tabular}

Tabla 4: Conexión en Músculos Esqueléticos

Fuente: Villée, 1975, p. 386-387, destaques del autor

Si se observan los recursos de la metafunción textual, los resultados del análisis se orientan a corroborar la hipótesis formulada sobre el género que instancia el Texto 1. En primer lugar, la IDENTIFICACIÓN, que permite hacer un seguimiento de entidades (MARTIN; ROSE, 2007), muestra (Tabla 5) que se producen en el texto una serie de elipsis que señalan la continuidad del tópico, representado por el músculo esquelético. La elipsis se marca con el

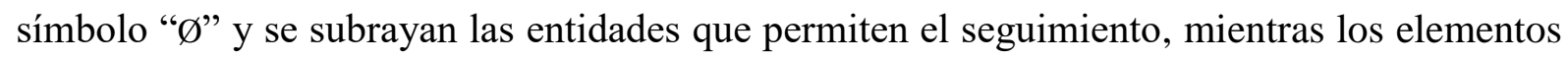
elididos se reponen entre corchetes simples.

\begin{tabular}{|c|c|}
\hline a. & Un músculo esquelético típico es una masa alargada de tejido, \\
\hline b. & formada por millones de fibras musculares individuales unidas por otras de tejido conjuntivo. \\
\hline c. & Toda la estructura $\varnothing$ [del músculo] está rodeada por una vaina elástica y lisa de tejido conjuntivo, \\
\hline d. & 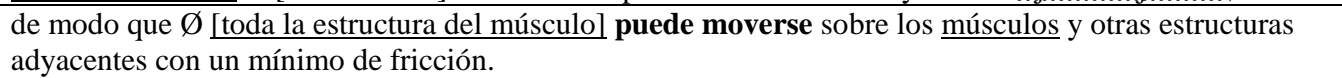 \\
\hline e. & Los dos extremos de un músculo están unidos, generalmente, a dos huesos distintos, \\
\hline f. & $\begin{array}{l}\text { aunque unos pocos } \varnothing\lceil\text { músculos] pasan de un hueso a la piel, o de una parte de la piel a otra, como en el } \\
\text { caso de los músculos faciales utilizados para la palabra y la expresión. }\end{array}$ \\
\hline g. & $\begin{array}{l}\text { El extremo } \emptyset[\text { del músculo] que permanece relativamente fijo cuando el músculo se contrae, se denomina } \\
\text { inserción de origen; }\end{array}$ \\
\hline h. & [[el Ø [extremo del músculo] que se mueve]] [se denomina] inserción terminal, \\
\hline i. & y la parte gruesa comprendida entre ambos [se denomina] vientre. \\
\hline j. & La inserción de origen del músculo bíceps está en el hombro, \\
\hline k. & y la Ø [inserción] terminal Ø Ødel músculo bíceps] [está] en el radio, en el antebrazo; \\
\hline 1. & cuando el bíceps se contrae, \\
\hline m. & el hombro permanece fijo \\
\hline n. & y el codo se flexiona. \\
\hline
\end{tabular}

Tabla 5: IDENTIFICACIÓN en Músculos Esqueléticos

Fuente: Villée, 1975, p. 386-387, destaques del autor

En segundo lugar, el análisis de los recursos de PERIODICIDAD (MOYANO, 2016; 2021) muestra que el método de desarrollo se realiza mediante los Temas de las diferentes 
figuras, que van desde el todo (músculo esquelético) hacia sus partes, siguiendo la relación de meronimia. El ángulo del campo seleccionado es, efectivamente, el músculo esquelético y sus partes (Tabla 6).

\begin{tabular}{|c|c|c|c|c|c|c|c|}
\hline & T txt & T interp & T exper marcado & $\begin{array}{l}\text { T exper } \\
\text { no marcado } \\
\end{array}$ & & Tema/Nuevo & Nuevo \\
\hline a & & & & $\begin{array}{l}\text { Un músculo } \\
\text { esquelético típico }\end{array}$ & es & & una masa alargada de tejido, \\
\hline b & & & & & formada & & $\begin{array}{l}\text { por millones de fibras musculares } \\
\text { individuales [[unidas por otras de } \\
\text { tejido conjuntivo]] }\end{array}$ \\
\hline c & & & & Toda la estructura & está & & $\begin{array}{l}\text { [[rodeada por una vaina elástica y } \\
\text { lisa de tejido conjuntivo]] }\end{array}$ \\
\hline d & $\begin{array}{l}\text { de } \\
\text { modo } \\
\text { que }\end{array}$ & & & [toda la estructura] & $\begin{array}{l}\text { puede moverse sobre los } \\
\text { músculos y otras estructuras } \\
\text { adyacentes }\end{array}$ & & con un mínimo de fricción. \\
\hline e & & & & $\begin{array}{l}\text { Los dos extremos de } \\
\text { un músculo }\end{array}$ & están & & $\begin{array}{l}\text { [[unidos, generalmente, a dos } \\
\text { huesos distintos]], }\end{array}$ \\
\hline$f$ & aunque & & & unos pocos & $\begin{array}{l}\text { pasan de un hueso a la piel, o } \\
\text { de una parte de la piel a otra, }\end{array}$ & & $\begin{array}{l}\text { como en el caso de los músculos } \\
\text { faciales [lutilizados para la palabra } \\
\text { y la expresión]]. }\end{array}$ \\
\hline g & & & & $\begin{array}{l}\text { El extremo [[que } \\
\text { permanece } \\
\text { relativamente fijo } \\
\text { cuando el músculo se } \\
\text { contrae]] }\end{array}$ & se denomina & & inserción de origen; \\
\hline $\mathrm{h}$ & & & & \begin{tabular}{|l}
{$[$ [elque se mueve]] } \\
\end{tabular} & $\begin{array}{l}\text { [se denomina] } \\
\end{array}$ & & inserción terminal, \\
\hline $\mathbf{i}$ & $y$ & & & $\begin{array}{l}\text { la parte gruesa } \\
\text { [[comprendida entre } \\
\text { ambos]] }\end{array}$ & [se denomina] & & vientre. \\
\hline $\mathbf{j}$ & & & & $\begin{array}{l}\text { La inserción de } \\
\text { origen del músculo } \\
\text { bíceps }\end{array}$ & está & & en el hombro, \\
\hline k & $y$ & & & la terminal & [está] en el radio, & & en el antebrazo; \\
\hline $\mathbf{I}$ & & & $\begin{array}{l}\text { cuando el bicees se } \\
\text { contrae. }\end{array}$ & & & & \\
\hline m & & & & el hombro & permanece & & fijo \\
\hline$n$ & $y$ & & & \begin{tabular}{|l|} 
el codo \\
\end{tabular} & se flexiona. & & \\
\hline
\end{tabular}

Tabla 6: PERIODICIDAD en Músculos Esqueléticos

Fuente: Villée, 1975, p. 386-387, destaques del autor

El único Tema marcado del texto está constituido por una cláusula dependiente (1). Esta decisión se toma a partir del criterio propuesto por Martin (1992b), quien basándose en Fries (1981) considera que una cláusula dependiente colocada antes de una independiente constituye un Tema marcado. Así, su función es establecer una transición en el texto: introduce la explicación del movimiento del brazo que produce la contracción del bíceps, presentado como ejemplo de músculo esquelético. Se trata, entonces, de un ejemplo de Tema bisagra (MOYANO, 2010b).

Finalmente, en cuanto a recursos de VALORACIÓN (MARTIN; WHITE, 2005; HOOD; MARTIN, 2005), es poco lo que este texto presenta. Sin embargo, como puede observarse en la Tabla 7, se muestran dos opciones de GRADUACIÓN (marcadas en gris) y una modalización (subrayada). Las graduaciones son la primera de foco (c) y la segunda de fuerza (d), mientras que la modalidad solo indica posibilidad de la actividad, no interacción de voces. 
c. de modo que puede moverse sobre los músculos y otras estructuras adyacentes con un mínimo de fricción.

d. Los dos extremos de un músculo están [[unidos, generalmente, a dos huesos distintos]],

Tabla 7: VALORACIÓN en Músculos Esqueléticos

Fuente: Villée, 1975, p. 387, destaques del autor

Todos los recursos señalados en este texto desde la perspectiva semántico-discursiva, con algunas miradas hacia el campo y hacia la lexicogramática, permiten comprobar que la hipótesis formulada era correcta: se trata de un informe composicional, como se muestra en la Tabla 8, con sus etapas obligatorias y fases optativas.

\begin{tabular}{|l|l|l|}
\hline ETAPAS & FASES & TEXTO \\
\hline Clasificación & Definición & $\begin{array}{l}\text { Un músculo esquelético típico es una masa alargada de tejido, formada } \\
\text { por millones de fibras musculares individuales unidas por otras de } \\
\text { tejido conjuntivo. }\end{array}$ \\
\hline \multirow{5}{*}{ Descripción } & $\begin{array}{l}\text { Coda la estructura está rodeada por una vaina elástica y lisa de tejido } \\
\text { conjuntivo, de modo que puede moverse sobre los músculos y otras } \\
\text { estructuras adyacentes con un mínimo de fricción. Los dos extremos de } \\
\text { un músculo están unidos, generalmente, a dos huesos distintos, aunque } \\
\text { unos pocos pasan de un hueso a la piel, o de una de una parte de la piel } \\
\text { a otra, como en el caso de los músculos faciales, utilizados para la } \\
\text { palabra y la expresión. }\end{array}$ \\
\cline { 2 - 3 } & Composición & $\begin{array}{l}\text { El extremo que permanece relativamente fijo cuando el músculo se } \\
\text { contrae, se denomina inserción de origen; el que se mueve, inserción } \\
\text { terminal, y la parte gruesa comprendida entre ambos, vientre (...). La } \\
\text { inserción de origen del músculo bíceps está en el hombro, y la terminal } \\
\text { en el radio, en el antebrazo; }\end{array}$ \\
\cline { 2 - 3 } & Función & $\begin{array}{l}\text { cuando el bíceps se contrae, el hombro permanece fijo y el codo se } \\
\text { flexiona. }\end{array}$ \\
\hline
\end{tabular}

Tabla 8: Estructura esquemática de Músculos Esqueléticos

Fuente: Villée, 1975, p. 386-387, destaques del autor

Con respecto al Texto 2 (Tabla 2), se había hipotetizado como instancia de algún tipo de explicación, dado el predominio de actividades de ocurrencia y la observación de relaciones causales. Pero para confirmar si esto es correcto, y en ese caso, de qué tipo de explicación se trata, hace falta más análisis.

En principio, el texto comienza con un complejo clausular que constituye un muy breve informe composicional de las Chlamydomonas (Tabla 9). Se trata de un fragmento que, observado desde el campo, presenta una perspectiva estática (DORAN; MARTIN, 2021). Predominan las actividades de estado (HAO, 2020), que ponen en relación de posesión dos entidades en la figura (a) y relacionan, en la figura (b), la entidad elidida con una cualidad. Se considera un informe y no una descripción porque se trata de caracterizar una clase de objeto. 


\begin{tabular}{|l|l|}
\hline a. & $\begin{array}{l}\text { Las Chlamydomonas, «que se encuentran en charcos, lagos y sobre la tierra húmeda,» poseen una célula } \\
\text { vegetativa [[que tiene dos flagelos]] // }\end{array}$ \\
\hline b. & y está [[protegida por una gruesa pared celulósica]]./// \\
\hline
\end{tabular}

Tabla 9: Informe composicional sobre de las Clamydomonas

Fuente: Villée, 1975, p. 192, destaques del autor.

A continuación, y observado el texto desde el campo, se presenta una perspectiva dinámica (DORAN; MARTIN, 2021). En la figura (c) se encuentra una actividad no momentada (unmomented activity), realizada por una figura de ocurrencia, que da cuenta de un proceso que va a desplegarse como secuencia de actividades: La célula puede reproducirse asexualmente por división. En la Tabla 10 se muestra el despliegue mediante actividades momentadas (momented activities), también figuras de ocurrencia, que van desde la cláusula (d) hasta la (g). Esto constituye, en el discurso, una secuencia de actividades (HAO, 2020).

\begin{tabular}{|l|l|}
\hline c. & La célula puede reproducirse asexualmente por división,// \\
\hline d. & formando de dos a ocho zoosporas dentro de la pared celulósica./// \\
\hline e. & Estas se liberan por rotura de la pared celular del progenitor// \\
\hline f. & y se alejan,// \\
\hline g. & constituyendo plantas independientes./// \\
\hline
\end{tabular}

Tabla 10: Despliegue de la primera actividad no momentada en Las Clamydomonas

Fuente: Villée, 1975, p. 192, destaques del autor.

Desde la perspectiva de la CONEXIÓn, se observa que los gerundios que realizan las actividades en las figuras (d) y (g) conllevan el significado de consecuencias. En la figura (e), se muestra una relación causal entre la metáfora gramatical rotura de la pared celular del progenitor y la liberación de las zoosporas, marcada por la preposición por. Puede decirse, entonces, que se trata en el campo de una secuencia de implicación (DORAN; MARTIN, 2021), que construye como género una explicación secuencial (MARTIN; ROSE, 2008).

De la misma manera, el texto continúa con otra actividad no momentada (figura (h)), realizada por una figura de ocurrencia, seguida de su despliegue mediante una secuencia de implicación (figuras (i) a (y)), como se puede observar en la Tabla 11. En algunos casos,

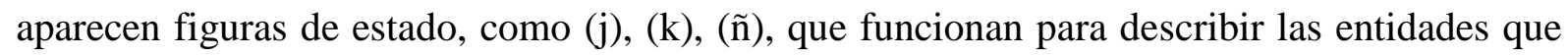
surgen de las actividades que se van presentando en el texto (gametas, cigota). 


\begin{tabular}{|l|l|}
\hline h. & Ocasionalmente, se realiza la reproducción sexual:// \\
\hline i. & la célula progenitora se divide para formar de ocho a treinta y dos células más pequeñas, las gametas,// \\
\hline j. & que se parecen a las zoosporas y a los individuos adultos// \\
\hline k. & pero son de menor tamaño./// \\
\hline l. & Dos de estas gametas se fusionan,// \\
\hline m. & comenzando por el extremo en [[el que nacen los flagelos]],// \\
\hline n. & para formar una cigota./// \\
\hline n. & Esta, al principio, tiene cuatro flagelos, dos de cada gameta,// \\
\hline o. & pero con el tiempo los pierde./// \\
\hline p. & La célula se redondea,// \\
\hline q. & segrega una gruesa pared celular// \\
\hline r. & y es capaz de sobrevivir largos períodos en un ambiente desfavorable./// \\
\hline s. & Cuando este se torna de nuevo favorable,// \\
\hline t. & la cigota sufre la meiosis// \\
\hline u. & y forma cuatro células./// \\
\hline v. & La pared celular se resquebraja// \\
\hline w. & dejando en libertad a las células,// \\
\hline x. & que emiten flagelos// \\
\hline y & y se transforman en plantas independientes./// \\
\hline
\end{tabular}

Tabla 11: Despliegue de la segunda actividad no momentada en Las Clamydomonas

Fuente: Villée, 1975, p. 192, destaques del autor.

En cuanto al sistema de CONEXIÓN, las opciones resultan menos claras. Sin embargo, ya está creada la expectativa del género. Hay una secuencia de actividades de ocurrencia que se vinculan por adición, pero para que las subsecuentes ocurran, se necesita que lo hagan las anteriores. De esta manera, las relaciones causales propias del género explicación secuencial se encuentran implícitas. No se trata de una secuencia de expectación (DORAN; MARTIN, 2021) sino de una secuencia de implicación que realiza una segunda explicación secuencial en el texto.

Otro sistema que interesa explorar aquí es el de PERIODICIDAD. Como se observa en la Tabla 12, la descripción de las Chlamydomonas funciona como macroTema, ya que anuncia las cadenas léxicas que se van a desplegar en el texto (la célula y sus subdivisiones para crear plantas independientes). Luego, hay dos hiperTemas: las dos actividades no momentadas (figuras (c) y (h)). Entre ambas, se despliegan las explicaciones, que presentan como Tema no marcado la célula y sus subdivisiones, hasta formar las plantas independientes. Es interesante señalar que la figura (h) es la única que presenta un Tema no marcado pospuesto, lo que destaca la reproducción sexual, que es la más evolucionada de las dos que dispone esta planta unicelular. Por otra parte, es la única figura que presenta un Tema interpersonal, que plantea la baja frecuencia del fenómeno, que contribuye a señalar su gradual evolución como especie. Finalmente, hay un Tema marcado que se destaca, realizado mediante una figura dependiente, que es la identificada como (s). En la figura anterior, el tiempo se detiene, y por lo tanto se detiene el proceso de reproducción. La figura (s) indica el momento en que se reactiva la 
reproducción y funciona como Tema bisagra, marcando un cambio en el texto (MOYANO, 2010b).

\begin{tabular}{|c|c|c|c|c|c|c|c|}
\hline & \begin{tabular}{|l|}
$T$ tat \\
\end{tabular} & T interp & T exper marcado & \begin{tabular}{|l|} 
T exper \\
no marcado
\end{tabular} & & Tema/Nuevo & Nuevo \\
\hline a & & & & $\begin{array}{l}\text { Las Chlamydomonas, } \\
\text { "que se encuentran en } \\
\text { charcos, lagos y sobre la } \\
\text { tierra húmeda," }\end{array}$ & poseen & & $\begin{array}{l}\text { una célula vegetativa } \\
\text { [Ique tiene dos flagelos]] }\end{array}$ \\
\hline b & $\mathrm{y}$ & & & [esa célula vegetativa] & está & & $\begin{array}{l}\text { protegida por una gruesa } \\
\text { pared celulósica. }\end{array}$ \\
\hline c & & & & La célula & puede reproducirse asexualmente & & por división, \\
\hline d & & & & [la célula] & formando de dos a ocho zoosporas & & $\begin{array}{l}\text { dentro de la pared } \\
\text { celulósica }\end{array}$ \\
\hline e & & & & Estas & se liberan & & $\begin{array}{l}\text { por rotura de la pared } \\
\text { celular del progenitor }\end{array}$ \\
\hline$f$ & $y$ & & & [estas] & se alejan, & & \\
\hline g & & & & [estas] & constituyendo & & plantos independientes. \\
\hline h & & Ocasionalmente, & & & se realiza & la reproducción sexual: & \\
\hline $\mathbf{i}$ & & & & la célula progenitora & se divide & & $\begin{array}{l}\text { para formar de ocho a } \\
\text { treinta y dos células más } \\
\text { pequeñas, las gametas, }\end{array}$ \\
\hline J & que & & & que (las gametas) & se parecen & & $\begin{array}{l}\text { a las zoosporos y a los } \\
\text { individuos aduitos }\end{array}$ \\
\hline k & pero & & & \begin{tabular}{|l|l} 
[las gametas] \\
\end{tabular} & son & & de menor tamaño. \\
\hline $\mathrm{I}$ & & & & \begin{tabular}{|l} 
Dos de estas gametas \\
\end{tabular} & se fusionan, & & \\
\hline m & & & & [dos de estas gametas] & comenzando & & $\begin{array}{l}\text { por el extremo en [[el que } \\
\text { nacen los flagelos]]. }\end{array}$ \\
\hline $\mathbf{n}$ & para & & & [dos de estas gametas] & formar & & una cigota. \\
\hline ก & & & & Esta, & al principio, tiene & & $\begin{array}{l}\text { cuatro flagelos, dos de } \\
\text { cada gameta, }\end{array}$ \\
\hline 0 & pero & & con el tiempo & [esta] & los pierde. & & \\
\hline $\mathbf{p}$ & & & & La célula & se redondea, & & \\
\hline 9 & & & & [la célula] & segrega & & una gruesa pared celular \\
\hline $\mathbf{r}$ & $y$ & & & [la célula] & es & & $\begin{array}{l}\text { capaz de sobrevivir largos } \\
\text { periados en un ambiente } \\
\text { desfavorable. }\end{array}$ \\
\hline s & & & $\begin{array}{l}\frac{\text { Cuando este se torna }}{\text { de nuevo favorable }} \\
\end{array}$ & & & & \\
\hline$t$ & & & & la cigota & sufre & & la meiosis \\
\hline u & $y$ & & & [la cigota] & forma & & cuatro células. \\
\hline $\mathbf{v}$ & & & & La pared celular & se resquebraja & & \\
\hline $\mathrm{w}$ & & & & [la pared celular] & dejando en libertad & & a las células, \\
\hline$x$ & que & & & que (las células) & emiten & & flagelos \\
\hline $\mathbf{y}$ & y & & & \begin{tabular}{|l|} 
[las células] \\
\end{tabular} & se transforman & & $\begin{array}{l}\text { en plantas } \\
\text { independientes. }\end{array}$ \\
\hline
\end{tabular}

Tabla 12: periodicidad en Las Chlamydomonas

Fuente: Villée, 1975, p. 192, destaques del autor.

Desde la perspectiva de la IDENTIFICACIÓN, se puede observar también en la Tabla 12 el seguimiento de entidades a través de la elipsis, que se muestra al introducir los Temas no marcados elididos entre corchetes simples, además del uso del pronombre estas (figura (e)) o

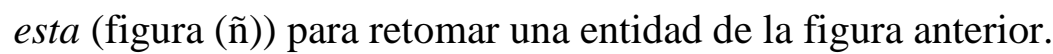

Finalmente, desde la VALORACión en el discurso (Tabla 13), se destacan solo algunos momentos en los que la apreciación ocupa un lugar en el texto, contribuyendo con la construcción del género. En la mayoría de los casos, se identifican en el Nuevo de unas pocas figuras signos de apreciación:composición (marcadas en gris), una de las cuales se encuentra graduada (subrayado). Asimismo, en la figura (k), se encuentra otro recurso de graduación (subrayado). Estas valoraciones dan cuenta de las características de las células que se generan por división (figuras (i) y (k)) o del tipo de pared celular que se origina en la nueva célula luego de perder los flagelos, rasgo que le permite mantenerse viva por largo tiempo en un ambiente que es valorado con signo de apreciación:reacción (subrayado doble), como 
desfavorable (figura (r)), rasgo del ambiente que detiene el proceso. En la figura (q) se destaca (subrayado punteado) un signo de apreciación:capacidad, que señala precisamente la condición de la célula de soportar las condiciones desfavorables para su reproducción. Luego, en (s) se cambia la valoración:reacción, a fin de explicar en qué momento se produce la continuidad del proceso reproductivo (subrayado doble). Como recurso destacado, se menciona el adjunto que se reconoció como Tema interpersonal (subrayado especial), que, como ya se señaló, indica la escasa frecuencia con que este tipo de reproducción más evolucionada se registra en las Chlamydomonas.

\begin{tabular}{|l|l|}
\hline h. & Ocasionalmente, se realiza la reproducción sexual:// \\
\hline i. & la célula progenitora se divide para formar de ocho a treinta y dos células más pequeñas, las gametas,// \\
\hline j. & que se parecen a las zoosporas y a los individuos adultos \\
\hline k. & pero son de menor tamaño. \\
\hline q. & segrega una gruesa pared celular// \\
\hline r. & y es capapaz de sobrevivir largos períodos en un ambiente desfavorable./// \\
\hline s. & Cuando este se torna de nuevo favorable,// \\
\hline t. & la cigota sufre la meiosis// \\
\hline u. & y forma cuatro células./// \\
\hline
\end{tabular}

Tabla 13: VALORACIÓN en Las Chlamydomonas

Fuente: Villée, 1975, p. 192, destaques del autor.

El texto se trata entonces de un macrogénero (Tabla 14). Se entiende por macrogénero una serie de textos adyacentes, cada uno de los cuales instancia un género, y que en conjunto logran un propósito mayor que el de cada uno (MARTIN, 1994; EGGINS; MARTIN, 2003; MARTIN; ROSE, 2008). El macrogénero que aquí se aborda está compuesto por un informe composicional de una planta unicelular, la Chlamydomona, y dos explicaciones secuenciales sucesivas, que dan cuenta de sus dos procesos alternativos de reproducción que, además de ejemplificar los tipos de reproducción existentes, muestran la evolución de la planta unicelular. Queda entonces confirmada la hipótesis generada sobre el género del texto, aunque se lo observa de mayor complejidad que lo esperado. 


\begin{tabular}{|c|c|c|}
\hline GÉNERO & ETAPAS & TEXTO \\
\hline $\begin{array}{c}\text { Informe } \\
\text { composicional }\end{array}$ & & $\begin{array}{l}\text { Las Chlamydomonas, que se encuentran en charcos, lagos y sobre la tierra } \\
\text { húmeda, poseen una célula vegetativa que tiene dos flagelos y está protegida } \\
\text { por una gruesa pared celulósica. }\end{array}$ \\
\hline \multicolumn{3}{|r|}{$\mathbf{X}$} \\
\hline \multirow[b]{2}{*}{$\begin{array}{l}\text { Explicación } \\
\text { secuencial } 1\end{array}$} & Anticipación & La célula puede reproducirse asexualmente por división, \\
\hline & Secuencia & $\begin{array}{l}\text { formando de dos a ocho zoosporas dentro de la pared celulósica. Estas se } \\
\text { liberan por rotura de la pared celular del progenitor y se alejan, constituyendo } \\
\text { plantas independientes. }\end{array}$ \\
\hline \\
\hline & Anticipación & Ocasionalmente, se realiza la reproducción sexual: \\
\hline $\begin{array}{l}\text { Explicación } \\
\text { secuencial } 2\end{array}$ & Secuencia & $\begin{array}{l}\text { la célula progenitora se divide para formar de ocho a treinta y dos células más } \\
\text { pequeñas, las gametas, que se parecen a las zoosporas y a los individuos } \\
\text { adultos, pero son de menor tamaño. Dos de estas gametas se fusionan, } \\
\text { comenzando por el extremo en el que nacen los flagelos, para formar una } \\
\text { cigota. Esta, al principio, tiene cuatro flagelos, dos de cada gameta, pero con el } \\
\text { tiempo los pierde. La célula se redondea, segrega una gruesa pared celular y es } \\
\text { capaz de sobrevivir largos períodos en un ambiente desfavorable. Cuando este } \\
\text { se torna de nuevo favorable, la cigota sufre la meiosis y forma cuatro células. } \\
\text { La pared celular se resquebraja dejando en libertad a las células, que emiten } \\
\text { flagelos y se transforman en plantas independientes. }\end{array}$ \\
\hline
\end{tabular}

Tabla 14: Estructura esquemática de Las Chlamydomonas

Fuente: Villée, 1975, p.: 192, destaques del autor.

En cuanto a las relaciones entre géneros, Martin propone (MARTIN, 1994; EGGINS; MARTIN, 2003) tomar las relaciones lógicas que Halliday (HALLIDAY; MATTHIESSEN, 2014) ofrece para la relación entre cláusulas en un complejo clausular. Así, en este macrogénero (Tabla 14), el informe composicional se ve ampliado (X) por las explicaciones secuenciales, que entre sí se vinculan por extensión (+), ya que la segunda ofrece una alternativa a la primera.

En síntesis, la metodología propuesta y validada en este trabajo sostiene varios pasos para el análisis de género:

1. Preparación del texto para el análisis mediante su segmentación en cláusulas.

2. Primera observación del registro.

3. Generación de hipótesis sobre el género que realiza el texto y de los pasos en que se despliega.

3. Análisis de los recursos lingüísticos relevantes, particularmente los semánticodiscursivos, para la construcción de significados en el texto, que permitirán comprobar las hipótesis sobre registro y género efectuadas en los pasos 2 y 3 .

Se trata de observar lo que Martin y Rose (2007) llaman primer plano (foregrounding) y co-articulación (co-articulation). El primer plano se refiere a los recursos que se prefieren por sobre otros para la construcción de significado en cada fase del texto; por co-articulación, cómo los sistemas semántico-discursivos seleccionados combinan sus recursos para producir 
un efecto en particular también fase a fase, lo que posteriormente Martin (2008) llamó acople (coupling).

Este análisis tiene que ser hecho desde una perspectiva trinocular: desde arriba, considerando el registro, desde alrededor, teniendo en cuenta los recursos de las diferentes metafunciones en el estrato semántico-discursivo y desde abajo, atendiendo, cuando fuera relevante, a los recursos de la lexicogramática que permitan su realización en ese estrato (HAO, 2020).

El análisis cualitativo del discurso efectuado de esta manera ofrece una doble perspectiva frente a cada texto: una perspectiva sinóptica, que lo observa como producto terminado, y una dinámica, que considera cómo los significados se van recontextualizando a medida que el texto se despliega (MARTIN, 2009). La perspectiva dinámica, entonces, permite mostrar cómo las elecciones adoptadas por el autor en un punto del discurso condicionan las elecciones siguientes (MARTIN; ROSE, 2007).

\section{Reflexiones finales}

En este trabajo se ha abordado una metodología para el análisis del discurso desde la teoría de género, registro y discurso de la LSF que puede orientar el trabajo de aplicación de los recursos teóricos desplegados en este marco. Asimismo, se procuró mostrar que es aplicable a los textos producidos en español, especialmente atendiendo a los desarrollos que hasta el momento se han elaborado sobre la descripción de esta lengua.

Se tomaron dos textos breves y sencillos de un manual de biología del nivel secundario, para mostrar cómo se puede caracterizar una instancia de un género y distinguirla de la instancia de otro. Se han analizado los recursos relevantes para la caracterización de cada género ejemplificado, así como se han señalado los procedimientos metodológicos básicos para trabajar en el análisis del discurso en el marco de la lingüística sistémicofuncional.

Como se ha dicho antes, la teoría está en movimiento. Seguramente hay que esperar nuevos desarrollos, especialmente en lo que hace a la caracterización del registro y su realización en la semántica-discursiva. Pero mientras tanto, es necesario más trabajo para la descripción de géneros y macrogéneros utilizados en los diferentes niveles educativos para contribuir a que los estudiantes evolucionen no solo en sus competencias en lectura y escritura de textos elaborados con lenguaje científico sino en la capacidad de construir conocimiento. Asimismo, es esperable que esta metodología pueda ser aplicada a textos de otros ámbitos 
sociales, a fin de contribuir con la comprensión de los procesos políticos, administrativos y comunicacionales.

En este sentido, este trabajo pretende constituir un aporte para que el desarrollo de análisis del discurso en español sea continuado. Es necesario avanzar con el camino ya iniciado, a fin de mostrar con mayor detalle que los recursos desarrollados para el inglés son aplicables al español así como en qué casos necesitan ser adaptados a esta lengua.

\section{REFERENCIAS}

BERNSTEIN, Basil. Pedagogy, Symbolic Control and Identity. London: Rowman and Littlefield, 2000.

CARLINO, Paula. Escribir, leer y aprender en la universidad: Una introducción a la alfabetización académica. Buenos Aires: Fondo de Cultura Económica, 2005.

CHRISTIE, Frances. Language Education throughout the School Years: A Functional Perspective. Malden: Wiley-Blackwell, 2012.

CHRISTIE, Frances; MARTIN, James R. (eds.). Genre and institutions: Social processes in the workplace and school. London: Contiuum, 1997.

DE SILVA JOYCE, Helen; FEEZ, Susan. Text-based language and literacy education: programming and methodology. Sydney: Phoenix Education, 2012.

DEREWIANKA, Beverly; JONES, Pauline. Teaching language in context. Oxford: Oxford University Press, 2012.

DORAN, Yaegan; MARTIN, James R. Field relations: Understanding scientific explanations. En: MATON, Karl; MARTIN, James R.; DORAN, Yaegan J. Teaching Science. Knowledge, Language, Pedagogy. London: Routledge, 2021, p. 105-133.

EGGINS, Suzanne; MARTIN, James R. El contexto como género: una perspectiva lingüístico-funcional. Revista Signos, Valparaíso, v. 36, n. 54, p.185-205, 2003.

FEEZ, Susan. Text-based Syllabus Design. New South Wales: AMES, 2006.

FRIES, P. On the status of Theme in English: Arguments from discourse. Forum Linguisticum, [s. l.] v. 6, n. 1, p. 1-38, 1981.

HALLIDAY, Michael A. K. Complementarities in Language. Beijing: The Commercial Press, 2008.

HALLIDAY, Michael A. K. Language in a Changing World. En: WEBSTER, Jonathan J. (ed.). On Language and Linguistics: Collected Works of M.A.K. Halliday. Vol. 3, London: Continuum, 2003 [1993]. p. 213-231. 
HALLIDAY, Michael A. K. The Notion of "Context" in Language Education. En: WEBSTER, Jonathan (Ed.). Language in Education: Collected Works of M.A.K. Halliday, Vol. 9. London: Continuum, 2007 [1991]. p. 269-290.

HALLIDAY, Michael A. K. Writing Science: Literacy and Discursive Power. En: WEBSTER, Jonathan (Ed.). The collected Works of M.A.K. Halliday. Vol. 5. The Language of Science. London: Continuum, 2004 [1993]. p. 199-235.

HALLIDAY, Michael A. K.; MATTHIESSEN, Christian M. I. M. An Introduction to Functional Grammar. 4th ed. London: Arnold, 2014.

HALLIDAY, Michael A. K; GREAVES, William S. Intonation in the Grammar of English. London: Equinox, 2008

HALLIDAY, Michael. A. K. El lenguaje como semiótica social. México: FCE, 1982 [1978].

HAO, Jing. Analysing Scientific Discourse from a Systemic Functional Perspective: A framework for exploring knowledge building in biology. London: Routledge, 2020.

HAO, Jing. Building taxonomies: A discourse semantic model of entities and dimensionality in biology. En: MATON, Karl; MARTIN, James R.; DORAN, Yaegan J. (Eds.). Teaching Science: Knowledge, Language, Pedagogy. London: Routledge, 2021.p. 134-161.

HAO, Jing. Construing biology: An ideational perspective, Unpublished $\mathrm{PhD}$ thesis, Department of Linguistics, University of Sydney, 2015.

HENRÍQUEZ, Rodrigo; CARMONA, Andrés; QUINTEROS, Alen; GARRIDO, Mabelin. Leer y escribir para aprender Historia: Secuencias ara la enseñanza y el aprendizaje del pensamiento histórico. Santiago: Ediciones UC, 2018.

HOOD, Susan; MARTIN, James R. Invocación de actitudes: El juego de la gradación de la valoración en el discurso. Revista Signos, Valparaíso, v. 38, n. 58, p. 195-220, 2005.

MARTIN James R.; ROSE, David. Working with discourse: Meaning beyond the clause. Australia: Bloomsbury, 2007.

MARTIN, James R. A contextual theory of language. En: COPE, Bill; KALANTZIS, Mary (Eds.). The Powers of Literacy: a genre approach to teaching literacy. London: Falmer, 1993. p. 116-136.

MARTIN, James R. Boomer dreaming: the texture of recolonisation in a lifestyle magazine. En: FOREY, G.; THOMPSON, G. (Eds.). Text-type and Texture. London, UK: Equinox Publishing, 2009. p. 252-284.

MARTIN, James R. Construing knowledge: a functional linguistic perspective. En: CRISTIE, Frances; MARTIN, James R. (Eds.). Language, Knowledge \& Pedagogy: functional linguistic and sociological perspectives. London: Continuum, 2007a. p. 34-64.

MARTIN, James R. English Text. System and Structure. Amsterdam: Benjamins, 1992a.

MARTIN, James R. Evolving Systemic Functional Linguistics: beyond the clause. Functional Linguistics, [s. l.], v. 1, n. 3, 2014. 
MARTIN, James R. Genre and field: social processes and knowledge structures in systemic functional semiotics. En: BARBARA, Leila; BERBER SARDINHA, Tony (Eds).

Proceedings of the 33rd International Systemic Functional Congress. São Paulo: PUCSP, 2007b. p. 1-35.

MARTIN, James R. Revisiting filed: Specialized knowledge in secondary school science and humanities discourse. En: MARTIN, James R.; MATON, Karl; DORAN, Yaegan J. (Eds). Accessing academic discourse: Systemic functional linguistics and legitimation code theory. London: Routledge, 2020. p. 114-148.

MARTIN, James R. Tenderness: realisation and instantiation in a Botswanan town. Odense Working Papers in Language and Communication, Denmark, Special Issue of Papers from 34th International Systemic Functional Congress edited by Nina Nørgaard, p. 30-62, 2008.

MARTIN, James R. Theme, method of development and existentiality: the price of reply. Occasional Papers in Systemic Linguistics, Nottingham, n. 6, p. 147-183, 1992 b.

MARTIN, James R., MATTHIESSEN, Christian M. I. M.; PAINTER, Claire. Deploying Functional Grammar. Beijing: The Commercial Press, 2010.

MARTIN, James R.; MATRUGLIO, Erika. Revisiting mode: Context in/dependency in Ancient History classroom discourse. En: MARTIN, James. R.; MATON, Karl; DORAN, Yaegan J. (Eds). Accessing academic discourse: Systemic functional linguistics and legitimation code theory. London: Routledge, 2020. p. 89-113.

MARTIN, James R.; QUIROZ, Beatriz; WANG, Ping; ZHU, Y. Systemic Functional Grammar: another step into the theory - grammatical description. Beijing: Higher Education Press, en prensa.

MARTIN, James R.; ROSE, David. Genre relations: Mapping culture. United Kingdom: Equinox, 2008.

MARTIN, James R.; WHITE, Peter. The language of evaluation: Appraisal in English. United Kingdom: Palgrave, 2005.

MARTIN, James. R. Macro-genres: The ecology of the page. Network, [s. l.], v. 21, n. 1, p. 29-52, 1994.

MATTHIESSEN, Christian M. I. M.; HALLIDAY, Michael A. K. Systemic functional grammar: A first step into the theory. China: Higher Education Press, 2009.

MOYANO, Estela I. (Coord). Aprender ciencias y humanidades: una cuestión de lectura y escritura: aportes para la construcción de un programa de inclusión social a través de la educación lingüística. Buenos Aires: Universidad Nacional de General Sarmiento, 2013.

MOYANO, Estela I. Aportes del análisis de género y discurso a los procesos de enseñanza y aprendizaje escolar: las ciencias biológicas y la historia. Discurso \& Sociedad, [s. l.], v. 4, n. 2, pp. 294-331, 2010a.

MOYANO, Estela I. Descripción de géneros para su enseñanza en un Programa de Escritura Académica. Hacia la Deconstrucción Conjunta. En: ÁVILA, N. Contribuciones multilingües 
a la investigación en escritura: Hacia un intercambio académico igualitario. Colorado: WAC Colostate, en prensa.

MOYANO, Estela I. El sistema de TEMA en español: una mirada discursiva sobre una cuestión controvertida. En: GHIO, Elsa; FERNÁNDEZ, María (Eds). El discurso en español y portugués: Estudios desde una perspectiva sistémico-funcional. Santa Fe: Universidad Nacional del Litoral, 2010b, pp. 39-87.

MOYANO, Estela I. Knowledge construction in Discussions of research articles in two disciplines in Spanish: The role of resources of APPRAISAL. Journal of Pragmatics, [s. l.], v. 139, p. 231-246, 2019.

MOYANO, Estela I. La Discusión en artículos de Microbiología: género, compromiso y construcción del conocimiento. Revista Onomázein, Santiago de Chile, número especial IX ALSFAL, p. 161-185, 2014.

MOYANO, Estela I. La escritura académica: una tarea interdisciplinaria a lo largo de la curricula universitaria. Revista Texturas, Santa Fe, v. 4, n. 4, p. 109-120, 2004.

MOYANO, Estela I. La función de Tema en español: sus medios de realización desde la perspectiva trinocular de la Lingüística Sistémico-Funcional. Revista Signos, Valparaíso, v. 54 n. 106, p. 487-517, 2021.

MOYANO, Estela I. La sección Discusión del artículo científico como género: Construcción del nuevo conocimiento y construcción del autor. Tesis doctoral, UBA, 2015.

MOYANO, Estela I. Theme in English and Spanish. Different means of realization for the same textual function. English Text Construction, [s. l.], v. 9, n. 1, p. 190-220, 2016.

NAVARRO, Federico. Estudios latinoamericanos de la escritura en educación superior y contextos profesionales: hacia la configuración de un campo disciplinar propio. Lenguas Modernas, Santiago de Chile, n. 50, p. 9-14, 2017.

OTEÍZA, Teresa. Escritura en la historia: Potencial de los recursos lingüísticos interpersonales e ideacionales para la construcción de la evidencia. Lenguas Modernas, Santiago de Chile, n.50, p. 193-224, 2017.

OTEÍZA, Teresa. Sistema de COMPROMISO en español escrito- dialogicidad en el campo del discurso de la historia. Boletín de Filología, Santiago de Chile, v. 3, n. 2, p. 799-819, 2021.

OTEÍZA, Teresa; HENRÍQUEZ, Rodrigo; CANELO, V. Language resources to negotiate historical thinking in history classroom interactions, Linguistics and Education, [s. l.], v. 47 pp. 1-15, 2018.

QUIROZ, Beatriz. La cláusula como movimiento interactivo: Una perspectiva semánticodiscursiva de la gramática interpersonal del español, DELTA: Documentação e Estudos em Linguística Teórica e Aplicada, São Paulo, v. 31, n. 1, p. 261-301, 2015.

QUIROZ, Beatriz. The verbal group. En: BARTLETT, Tom; O'GRADY, Gerard (Eds.). The Routledge Handbook of Systemic Functional Linguistics. London: Routledge, 2017. p. 301318. 
QUIROZ, Beatriz. Towards a systemic profile of the Spanish MOOD. Linguistics and the Human Sciences, [s. l.], v. 4, n. 1, p. 31-65, 2008.

ROSE, David; MARTIN, James R. Learning to Write, Reading to Learn: Genre, Knowledge and Pedagogy in the Sydney School. United Kingdom: Equinox, 2012.

VIDAL LIZAMA, Margarita. Aproximación al ensayo académico como género de formación en Ciencias Sociales: el caso de Sociología DELTA: Documentação e Estudos em Linguística Teórica e Aplicada, São Paulo, v. 36, n. 4, p. 1-26, 2020.

VIDAL LIZAMA, Margarita; LEIVA, Natalia. Aproximación discursiva a la 'solemne' en Derecho desde la LSF: una aplicación del sistema de figura'. Literatura y Lingüística, Santiago de Chile, en prensa.

VIDAL LIZAMA, Margarita; MONTES, Soledad. Escritura académica en la formación de estudiantes de Arte: continuidades y cambios a lo largo de la carrera. En: MOYANO, Estela I.; VIDAL LIZAMA, Margarita (Eds.). Centros y Programas de Escritura en América Latina: opciones teóricas y pedagógicas para la alfabetización académica. Colorado: The WAC Clearinghouse, en prensa.

VILEÉ, Claude. Biología. Buenos Aires: EUDEBA, 1975.

Artigo submetido em: 25 abr. 2021

Aceito para publicação em: 23 jun. 2021

DOI: http://dx.doi.org/10.22456/2238-8915.113374 